PROCEEDINGS OF THE

AMERICAN MATHEMATICAL SOCIETY

Volume 128, Number 2, Pages 541-545

$\mathrm{S}$ 0002-9939(99)05035-2

Article electronically published on July 8, 1999

\title{
SOLVING THE $p$-LAPLACIAN ON MANIFOLDS
}

\author{
MARC TROYANOV
}

(Communicated by Peter Li)

\begin{abstract}
We prove in this paper that the equation $\Delta_{p} u+h=0$ on a $p$-hyperbolic manifold $M$ has a solution with $p$-integrable gradient for any bounded measurable function $h: M \rightarrow \mathbb{R}$ with compact support.
\end{abstract}

\section{INTRODUCTION}

The $p$-Laplacian of a function $f$ on a connected oriented Riemannian manifold without boundary $M$ is defined by $\Delta_{p} f=\operatorname{div}\left(|\nabla f|^{p-2} \nabla f\right)$; it is the Euler-Lagrange operator associated with the functional $\int_{M}|\nabla f|^{p}$.

A function $u \in W_{l o c}^{1, p}(M)$ is said to be a weak solution to the equation

$$
\Delta_{p} u+h=0
$$

if for all $\psi \in C_{0}^{1}(M)$ one has

$$
\int_{M}\left\langle|\nabla u|^{p-2} \nabla u, \nabla \psi\right\rangle=\int_{M} h \psi .
$$

We introduce the $p$-Dirichlet space $\mathcal{L}^{1, p}(M)$ of functions $u \in W_{l o c}^{1, p}(M)$ admitting a weak gradient such that $\int_{M}\|\nabla u\|^{p}<\infty$.

In [2], the following result has been proved:

Theorem 1. Suppose that $M$ is p-parabolic, and let $h \in L^{1}(M)$ be a function such that $\int_{M} h \neq 0$. Then (1) has no weak solution $u \in \mathcal{L}^{1, p}(M)$.

The goal of this paper is to prove the following result in the converse direction.

Theorem 2. Suppose that $M$ is a p-hyperbolic manifold $(1<p<\infty)$ and that $h \in L^{\infty}(M)$ has compact support. Then (1) has a weak solution $u \in \mathcal{L}^{1, p}(M)$. Moreover $u$ is of class $C^{1, \alpha}$ on each compact set (where $\alpha \in(0,1)$ may depend on the compact set).

The notion of $p$-hyperbolic and $p$-parabolic manifolds will be recalled below (see also [6]). As an example, the euclidean space $\mathbb{R}^{n}$ is $p$-hyperbolic if and only if $p<n$.

Remark. If $M=\mathbb{R}^{n}$ with $1<p<n$ and $h \geq 0$, then equation (1) (and in fact a more general eigenvalue problem) is solved in [1].

Received by the editors April 6, 1998.

1991 Mathematics Subject Classification. Primary 31C15, 31C12, 31C45; Secondary 53C20.

Key words and phrases. Differential geometry, potential theory, non linear partial differential equations.

(C)1999 American Mathematical Society 


\section{Preliminaries on $p$-Hyperbolicity}

Definition. Let $(M, g)$ be a connected Riemannian manifold, and $K \subset M$ a compact set. For $1<p<\infty$, the $p$-capacity of $K$ is defined by

$$
\operatorname{Cap}_{p}(K):=\inf \left\{\int_{M}|\nabla u|^{p}: u \in C_{0}^{1}(M), u \geq 1 \text { on } K\right\} .
$$

The manifold $M$ is said to be $p$-parabolic if $\operatorname{Cap}_{p}(K)=0$ for all compact subsets $K \subset M$ and $p$-hyperbolic otherwise. It is a well known fact that, in a $p$-hyperbolic manifold, the $p$-capacity of any compact set with non empty interior is always positive (see e.g. [6]).

Let $D \subset M$ be a non empty bounded domain. We introduce the Banach space $E^{p}=E^{p}(D, M)$ of functions $u \in W_{l o c}^{1, p}(M)$ such that

$$
\|u\|_{E}^{p}:=\int_{D}|u|^{p} d x+\int_{M}|\nabla u|^{p} d x<\infty .
$$

We denote by $E_{0}^{p}$ the closure of $C_{0}^{1}(M)$ in $E^{p}$.

Lemma 1. If $M$ is p-parabolic, then $1 \in E_{0}^{p}$.

Proof. By hypothesis $\operatorname{Cap}_{p}(\bar{D})=0$; hence for all $\epsilon>0$, there exists a function $u \in C_{0}^{1}(M)$ such that $u \equiv 1$ on $D$ and $\int_{M}|\nabla u|^{p} d x<\epsilon$. Thus we have

$$
\|1-u\|_{E}^{p}:=\int_{D}|1-u|^{p} d x+\int_{M}|\nabla u|^{p} d x=\int_{M}|\nabla u|^{p} d x \leq \epsilon .
$$

It follows that $1 \in E_{0}^{p}$.

The next lemma is the well known Poincaré inequality.

Lemma 2. Let $D$ be any bounded regular domain in a Riemannian maniflold $M$ and $1 \leq p<\infty$. Then there exists a constant $A$ such that

$$
\left(\int_{D}\left|u-u_{D}\right|^{p} d x\right)^{1 / p} \leq A\left(\int_{D}|\nabla u|^{p} d x\right)^{1 / p}
$$

for all $u \in W_{\text {loc }}^{1, p}(M)$, where $u_{D}=\frac{1}{\operatorname{vol}(D)} \int_{D} u d x$ is the mean value of $u$ on $D$.

A reference is [3, Lemma 3.8].

Combining this lemma with Hölder's (or Jensen's) inequality, we obtain

Corollary 1. There exists a constant $c=c_{D}$ such that

$$
\int_{D}\left|u-u_{D}\right| d x \leq c_{D}\left(\int_{M}|\nabla u|^{p} d x\right)^{1 / p}
$$

for all $u \in W_{l o c}^{1, p}(M)$.

Proposition 1. Suppose that $M$ is p-hyperbolic and let $D \subset M$ be as in Lemma 2. Then there exists a constant $C_{1}$ such that for all $u \in E_{0}^{p}$

$$
\int_{D}|u| d x \leq C_{1}\left(\int_{M}|\nabla u|^{p} d x\right)^{1 / p} .
$$


Proof. Suppose that such a constant does not exist. Then for all $\varepsilon>0$ it is possible to find a function $u \in E_{0}^{p}$ such that

$$
\int_{D}|u| d x=\operatorname{vol}(D) \quad \text { and } \quad\|\nabla u\|_{L^{p}(M)} \leq \varepsilon .
$$

We may also assume $u \geq 0$ (else replace $u$ by $|u|$ ). From Corollary 1 one gets

$$
\int_{D}|u-1| d x \leq c_{D} \varepsilon
$$

Let us now choose a ball $B \subset \subset D$ and a function $\psi \in C_{0}^{1}(M)$ such that $0 \leq \psi \leq \frac{1}{2}$, $\operatorname{supp}(\psi) \subset D$ and $\psi \equiv \frac{1}{2}$ on $B$, and define the function $v \in E_{0}^{p}$ by $v=2 \max \{u ; \psi\}$.

Observe first that $v \geq 1$ on $B$, and define the sets

$$
A:=\{x \in D \mid \psi(x) \geq u(x)\} \quad \text { and } \quad A^{\prime}:=\left\{x \in D|| u(x)-1 \mid \geq \frac{1}{2}\right\} .
$$

We have $A \subset A^{\prime}$ and by (3) we have $\frac{1}{2} \operatorname{vol}\left(A^{\prime}\right) \leq c_{D} \varepsilon$; thus

$$
\operatorname{vol}(A) \leq 2 c_{D} \varepsilon
$$

Now we have almost everywhere

$$
\nabla v=\left\{\begin{array}{lll}
2 \nabla u & \text { on } & M \backslash A, \\
2 \nabla \psi & \text { on } & A
\end{array}\right.
$$

in particular

$$
|\nabla v| \leq 2|\nabla u|+2 \chi_{A}|\nabla \psi| \quad \text { a.e. }
$$

from which one deduces

$$
\|\nabla v\|_{L^{p}(M)} \leq 2\|\nabla u\|_{L^{p}(M)}+2 \sup |\nabla \psi|(\operatorname{vol}(A))^{1 / p} .
$$

From (4) and (5) one obtains

$$
\|\nabla v\|_{L^{p}(M)} \leq\left(2 \varepsilon+2 \sup |\nabla \psi|\left(2 c_{D} \varepsilon\right)^{1 / p}\right) .
$$

Since $v \geq 1$ on $B$ and $\varepsilon$ is arbitrary, one deduces that $\operatorname{Cap}_{p}(B)=0$, which contradicts the fact that $M$ is $p$-hyperbolic.

We may sum up our results so far in

Theorem 3. The following conditions are equivalent:

(a) $M$ is p-hyperbolic;

(b) There exists a constant $C_{2}$ such that for all $u \in E_{0}^{p}$ one has

$$
\|u\|_{L^{p}(D)} \leq C_{2} \cdot\|\nabla u\|_{L^{p}(M)} ;
$$

(c) $1 \notin E_{0}^{p}$.

Proof. The implication (b) $\Rightarrow(\mathrm{c})$ is obvious and (c) $\Rightarrow$ (a) is Lemma 1 . 
Let us write $u$ as $u=\left(u-u_{D}\right)+u_{D}$; using Proposition 1 and Lemma 2, we see that

$$
\begin{aligned}
\|u\|_{L^{p}(D)} & \leq\left\|u-u_{D}\right\|_{L^{p}(D)}+\left\|u_{D}\right\|_{L^{p}(D)} \\
& \leq A\left(\int_{D}|\nabla u|^{p} d x\right)^{1 / p}+(\operatorname{Vol}(D))^{1 / p}\left|u_{D}\right| \\
& \leq A\left(\int_{D}|\nabla u|^{p} d x\right)^{1 / p}+(\operatorname{Vol}(D))^{(1-p) / p} \int_{D}|u| d x \\
& \leq A\left(\int_{D}|\nabla u|^{p} d x\right)^{1 / p}+(\operatorname{Vol}(D))^{(1-p) / p} C_{1}\left(\int_{M}|\nabla u|^{p} d x\right)^{1 / p} \\
& \leq C_{2}\left(\int_{M}|\nabla u|^{p} d x\right)^{1 / p} .
\end{aligned}
$$

This proves $(\mathrm{a}) \Rightarrow(\mathrm{b})$.

\section{Proof of Theorem 2}

We first choose a regular bounded domain $D \subset M$ such that $\operatorname{supp}(h) \subset D$. We then define a functional $\mathcal{J}: E_{0}^{p} \rightarrow \mathbb{R}$ by

$$
\mathcal{J}(u)=\frac{1}{p}\left(\int_{M}|\nabla u|^{p} d x\right)-\int_{M} h u d x .
$$

The manifold $M$ beeing $p$-hyperbolic, we have

$$
\begin{aligned}
\mathcal{J}(u) & \geq \frac{1}{p}\|\nabla u\|_{L^{p}(M)}^{p}-\left|\int_{M} h u d x\right| \\
& \geq \frac{1}{p}\|\nabla u\|_{L^{p}(M)}^{p}-\|h\|_{L^{\infty}} \cdot\|u\|_{L^{1}(D)} \\
& \geq \frac{1}{p}\|\nabla u\|_{L^{p}(M)}^{p}-C_{1}\|h\|_{L^{\infty}} \cdot\|\nabla u\|_{L^{p}(M)},
\end{aligned}
$$

where $C_{1}$ is the constant of Proposition 1. Since the function $g(x)=|x|^{p}-a x$ of the real variable $x$ is bounded below, we conclude that the functional $\mathcal{J}$ is bounded below on the space $E_{0}^{p}$.

Set $m:=\inf \left\{\mathcal{J}(u) \mid u \in E_{0}^{p}\right\}$, and let $\left\{u_{i}\right\} \subset E_{0}^{p}$ be a minimizing sequence for $\mathcal{J}$ (i.e. $\mathcal{J}\left(u_{i}\right) \rightarrow m$ ). Then from the inequality above, one deduces that $\left\{u_{i}\right\}$ is a bounded sequence in $E_{0}^{p}$. Since $E_{0}^{p}$ is a reflexive Banach space, this sequence contains a weakly convergent subsequence (still denoted by $\left\{u_{i}\right\}$ ). Let us denote by $u^{*}$ the weak limit of $\left\{u_{i}\right\}$. By the compactness of the embedding $E_{0}^{p} \subset L^{1}(D)$, we may assume that $\left\{u_{i}\right\}$ converges strongly in $L^{1}(D)$, in particular

$$
\int_{D} h u_{i} \rightarrow \int_{D} h u^{*}
$$

By Theorem 3, $\|\nabla u\|_{L^{p}(M)}$ is an equivalent norm on $E_{0}^{p}$; hence by the weak lower semi-continuity of the norm on $E_{0}^{p}$ we have

$$
\left\|\nabla u^{*}\right\|_{L^{p}(M)} \leq \lim _{i \rightarrow \infty} \inf \left\|\nabla u_{i}\right\|_{L^{p}(M)} .
$$


From (6) and (7) one deduces that $\mathcal{J}\left(u^{*}\right) \leq \lim _{i \rightarrow \infty} \inf \mathcal{J}\left(u_{i}\right)=m$; hence $\mathcal{J}\left(u^{*}\right)=m$. By the usual arguments from variational calculus, one deduces that $u^{*}$ is a weak solution to (1).

The $C^{1, \alpha}$ regularity follows from Theorem 1 in [5].

Remark. We have in fact solved (1) in the space $E_{0}^{p} \subset \mathcal{L}^{1, p}(M)$.

\section{REFERENCES}

[1] P. Drábek, Nonlinear eigenvalue problem for p-Laplacian in $\mathbb{R}^{n}$, Math. Nachr. 173 (1995), 131-139. MR 96b:35064

[2] V. Gol'dshtein and M. Troyanov, Sur la non résolubilité du p-laplacien sur $\mathbb{R}^{n}, \mathrm{C}$. R. Acad. Sci. Paris 326 (1998), 1185-1187. CMP 99:02

[3] E. Hebey, Sobolev spaces on Riemannian manifolds, Springer Lect. Notes in Math. 1635. CMP 98:04

[4] J. Heinonen, T. Kilpeläinen, and O. Martio, Nonlinear potential theory of degenerate elliptic equations, Oxford Math. Monographs (1993). MR 94e:31003

[5] P. Tolksdorf, Regularity for a more general class of quasilinear elliptic equations, J. Diff. Equations 51 (1984), 126-150. MR 85g:35047

[6] M. Troyanov, Parabolicity of manifolds, préprint EPFL, 1997.

Départment de Mathématiques, Ecole Polytechnique Fédérale de Lausanne, 1015 LaUsanne, Switzerland

E-mail address: troyanov@math.epfl.ch 